was drawn out at the anterior angle, which accounted for death in that instance, as the patient on the fifth day pushed the dressings back in order to free his eyc, and thus exposed the angle of the wound. $\mathrm{He}$ was found with the wound unprotected and the cerebrospinal fluid flowing out of the opening left by the drain. Forty-eight hours after the exposure he died of septic meningitis. The packing was usually removed on the third day.

Of the recent routes advised the most attractive appears to be that of Harvey Cushing. ${ }^{10}$ The opening is on a lower level than in the Hartley-Krause operation, which removes the obstruction offered by the zygoma. A cursory description of the operation may be given as follows: A small $\cap$-shaped incision, base directed downward, is made between the eye and the ear, the upper portion of the incision being one $\mathrm{cm}$. above the center of the zygoma, the lower points of it being two $\mathrm{cm}$. below. The zygoma is divided at each extremity and turned down with the flap and the incision then carried through the temporal muscle down to the temporal bone. The attachment to the bone is next scraped away with the periosteum, and in this way the lower portion of the temporal fossa of the skull, as far down as the attachment of the external pterygoid muscle, bolow the infratemporal crest, is well cxposed. A gouge or small trephine is then used for removing a portion of the bone. The opening should then be cnlarged with a rongeur until it is about $3 \mathrm{~cm}$. in diameter. The middle meningeal artery can now be seen, and the work should be carried on anterior to it.

The dura is next elevated, and this can be done almost bioodlessly, to the foramen ovale, and the elevation extorded forward to the foramen rotundum. The commissure of the under layer of the dura is then divided and the dura gently raised from the ganglion well back on to the sensory root. Before dividing the branches the ganglion is elevated and freed from its attachment to the under layer of the dura. The ganglion is then separated from its attachment to the first branch, and this must be done gently so as to avoid the cavernous sinus. The three peripheral branches are now elcvated with blunt hooks and divided close to the foramina. The ganglion is grasped with a forceps and evulsed. The bone. fascia and muscles are replaced and held in position by sutures. No drainage is used. While we have not performed this operation except on the cadaver, it appears to have the following advantages: 1 . Smaller incision; 2. less extracranial hemorrhage and less bleeding from thie venæ Santorini, and the probable avoidance of the middle meningeal artery ; 3 , ready access to the ganglion without cerebral compression, and 4 , if drainage is necessary it will be at the most dependent portion of the cranial cavity.

( To be continued.)

10. Jovr. A. M. A., April 28, 1900.

The Right of Privacy.-The Philadelphia Press has recently called attention to a decision of an English law court that is of great importance to physicians. According to this English decision (which would probably hold good in most Ameriean courts), a regular physician has no remedy if he preseribes a proprietary medicine, and the owner of the medicine publishes that fact in his advertisement. It is just as well for physicians to understand this matter. The market is flooded with proprietary drugs, and not a few of these are of distinct value. The custom of prescribing them is quite common, and is increasing.-Phila. Med. rour.

\section{THE SURGERY OF RICKETS.*}

HENRY LING TAYLOR, M.D.

Professor of Orthopedic Surgery, New York Post-Graduate Mredical School and Hospital. NEW YORK CITY.

Out of 42,124 new cases applying for treatment in the ten years, 1890-1899, at the orthopedic clinic of the Hospital for the Ruptured and Crippled, 6.583, or over 15 per cent., suffered from rickets or the deformities most frequently caused by rickets, such as bow-legs, knock-knees and pigeon breast. While it would be erroneous to class all cases of bow-legs and knock-knees as rhachitic, in the class and grade of cases applying at the clinic the large majority are due to this causc. Making allowance for a considerable number of patients with varicose veins and other purely medical or surgical affections, it is safe to say that rhachitic paticnts form nearly one-fifth of the orthopedic cases at this clinic.

Of the total number of rhachitics about one-half came on account of bow-legs, over one-quarter for knockknees and the remainder for anterior tibial curves. rhachitic spine, pigeon breast and other deformities, or for the rhachitic condition. $\Lambda$ large majority of the cases were under 5 years of age. As already hinted many of the slighter and a few of the severe cases of bow-legs and knock-knees in young ehildren are due to other causes. When arising in adolescence they seem usually to be due to static conditions, since the general symptoms of rickets are absent. Most cases of lateral curvature and of coxa vara are probably not rhachitic, though many of the early cases are. Very many of the milder cases of bow-legs and knock-knees, whether rhachitic or not, recover without special surgical treatment, and in these, incleed in all: a roborant régime, aiming to improve assimilation and nutrition, is indicated. Plenty of sunlight and fresh air, a nourishing diet, containing fresh milk, eggs, fresh meat or meat juice, gravy, digestible fats and fresh fruits are of the greatest importance. Cod-liver oil and iron are often valuable: and good results are reported from the use of phosphorus and of the lactophosphates.

The classes in New York among which rickets is most common are the Italians, the negroes and the Russian and Polish Jews, and it is these people who most notoriously sin against hygienic laws. It is common for them to feed their babies on condensed milk and artificial foods, with additions of tea, coffee, wine or beer, and cake and other solids from the table. Their babies are often kept on the breast $u$ p to the age of two rears or more, eren if the mother has again become pregnant. Nevertheless rickets is not confined to the poor, the ignorant, nor to the city dweller. In improper diet is probably the most potent cause.

It should be remembered that the malnutrition of rickets profoundly affects all the tissues, including the muscles, nerves and glands, not merely the bones; the sweating, the weakness, the bronchial and intestinal catarrh are evidences of a widespread nutritional vice which must be corrected if surgery is to succeed. The blood must be cnriched and the body generously nourished. A change of air, especially a trip to the seashore and salt-water bathing are often of considerable value.

The earlier stigmata, and even the earlier deformities, such as the epiphyseal swellings, beaded ribs, square forehead, large fontanelles and protuberant abdomen are often unnoticed or made light of by the parents, * Read at the Fifty-third Annual Meeting of the American
Hedical Association, in the Section on Surgery and Anatomy, and approved for publication by the Executive Committee: Drs. H. 0 . Walker, A. J. Ochsner and DeForest Willard. 
who may first be disturbed by marked deformities of the back or limbs or by the child's weakness or inability to stand or walk at eighteen months or two years of age. This slowness in walking is often a fortunate circumstance and one much to be encouraged, since at this early stage walking could only result in a rapid increase of deformity. In the stage of bony softening then it is often of primary importance to keep the child off his feet or to limit walking and standing, whether the predominant deformity be bow-legs, knock-knees or kyphosis. In the latter affection even sitting may be productive of harm, and the prone position for some hours a day may be indicated. The generous use of the perambulator serves the double purpose of bringing the child into the fresh air and keeping him off his feet. Particularly in these non-ambulant cases are massage. frictions with cold water or diluted alcohol and manipulations of the trunk and extremities of service, though they are worthless for the correction of serious deformities. In mild ambulant cases it is often well to build up the outer edge of the shoe in bow-legs and the inner edge in knock-knees, in addition to the friction manipulations and exercises. As the child becomes older and more active, passing a larger part of its time in the open air and better able to assimilate an ordinary mixed diet, the nutritional vice usually subsides, either with or without treatment, and the various deformities of the back and limbs, unless very severe, tend to improve; many of the milder cases are completely corrected. Bow-legs improve more than knock-knees, or anterior curves of the tibia, and rhachitic kyphosis improves oftener than rhachitic scoliosis and pigeon breast.

Just a word as to rhachitic spine, which it is exceedingly important and also exceedingly easy to differentiate from spondylitis. In rhachitic spine the kyphos is rounded, involving many vertebræ, and may be partly or wholly obliterated by lifting the legs and pelvis, the child being prone.

In Pott's disease, in the active stage, there is a sharp knuckle at some point of the line of the spinous processes with muscular rigidity. The characteristic pains are at the sides or in front, and it often hurts the child to be lifted. There are no characteristic pains in rhachitic spine. In spondylitis the children are usually older and are nearly always able to walk. They cry at night and have a characteristic attitude in walking, standing and stooping.

The treatment of rhachitic spine is usually the treatment of rickets with the addition of recumbency or procumbency and massage. Apparatus is rarelv needed. As the rickets improve the back becomes stronger, and a perfect recovery may usually be expected. Infantile scoliosis due to rickets requires similar treatment, with some additions, but is much more intractable. In pigeon-breast obstructions to the air passages are to be removed and pulmonary gymnastics employed.

If at one and a half to three years of age a child presents a fairly severe bow-leg or knock-knee, braces exerting a constant correcting leverage on the distorted bones should be worn. In most cases of bow-legs the Knight brace, taking its fixed points at the inner side of the os calcis and the internal condyle, and drawing the tibia over by a lacing to the bar on the inner side of the leg, answers the requirements. If, however, the femur as well as the tibia is bowed, a stiff apparatus should extend to the groin, and the knee and lower thigh be included in the lacing.

For knock-knees in its milder form the stiff Thomas knock-knee splint, making pressure over the outer side of the heel, great trochanter and counterpressure against the inner side of the knee, gives excellent results.

When the bones are too hard or the deformity too great to be corrected by splints, which is often the case after three or four years of age, an operation is to be considered. The classical operations are osteotomy and osteoclasis. Osteoclasis may be manual or instrumental. Manual osteoclasis is applicable in very voung cases, when deformity is severe enough to require interference; in those and in all young cases where the rickets is still active, the after-treatment and general anti-rhachitic measures should be strictly attended to. Rapid osteoclasis, as described by Dr. Wallace Blanchard of Chicago, is an excellent operation arid leaves little to be desired. The older methods are less neat and precise than osteotomy, and the latter operation, as improved under the modern surgical technic, is preferred by most New York surgeons. While bones have been broken and cut from the time of the ancients, it was not until after the adoption of asepsis in surgery that the cutting operation became a standard one.

At present the osteotome, or double-faced chisel, has displaced the saw, and the operation may be done subcutaneously, the operator never seeing the bone. Under proper technic the operation may be compared to subcutaneous tenotomy in the simplicity of the method and the certainty and precision of the results. Osteotomies for knock-knee involving the knee joint are unsatisfactory and unnecessary and have been abandoned.

For a knock-knee the femur is chisled a finger's breadth above the internal condyle (McEwen), or above the external condyle (McCormack). The only appliances needed are an osteotome with a cutting edge threeeighths of an inch wide and a sandbag. The patient having been properly prepared and anesthetized, an assistantholds the knee firmly against the sandbag in the semiflexed position and with the outer or the inner side uppermost. The operator having selected the point of entrance, and bearing clearly in mind the anatomy of the parts, guards the osteotome with his thumb and forces it down to the bone, keeping the edge parallel with its long axis; having penetrated the periosteum, the osteotome is turned 90 degrees, so that its edge lies across the long axis of the bone, and is driven nearly through it by rapid strokes of the mallet, the position of the osteotome being slightly changed in the plane of the section to avoid wedging and to complete the cut. When the bone, including the outer shell, has been about three-quarters divided, the osteotome is withdrawn, and the fracture completed by hand with a tangible and audible shock.

The deformity is then slightly overcorrected, good apposition being assured by unsevered portions of the periosteum. Though no Esmarch has been applied the bleeding is usually trifling, often but a few drops. The limb is quickly cleaned up, a sterile gauze and cotton dressing applied, and over this a plaster-of-paris spica from the axilla to the toes. This dressing is usually not disturbed for six weeks, and may be followed by a 'Thomas knock-knee splint or a plaster splint from the ankle to the groin. Suppuration or other accidents are very rare, but unless great pains are taken the deformity may be incompletely corrected or may recur from imperfect application or too early relaxation of the post-operative treatment. In young children it is not safe to dispense with splints for six months or more.

In bow-legs the greatest bowing is oftenest at the junction of the middle and lower thirds of the tibia, and it is here that the section is usually made. The opera- 
tion is strictly similar to the operation for knock-knees, but simpler, as the tibia is nearer the surface. The bone is attacked on its subcutaneous surface, the shape of the bone and its relation to surrounding parts being borne in mind. The fracture is completed by hand as before. It is necessary to remember that, in cases of bow-legs severe enough for operation, there is a considerable inward twist of the tibia, which causes marked inversion of the feet. When the knees point forward the feet usually turn in 15 to 30 degrees. In order to correct this deformity it is necessary to break or sharply bend the fibula after division of the tibia, to overcorrect the bowing; and also to rotate the lower fragment outward. This is not always easy, and is frequently neglected in practice, and I have in some instances seen poor results from the neglect of this precaution. For bow-legs the plaster splint should reach from the toes to the groin, and if firmly adjusted the patient may walk at the end of a week; in the hands of a non-expert it is better to keep the child recumbent for a month. Careful attention to splinting for some months after the operation is essential to a successful result.

In the past ten years 428 cases of bow-legs, knockknees and anterior tibial curves in children were submitted to operation at the Hospital for the Ruptured and Crippled. In most of these the operations were double, and in several instances multiple. In 367 cases one or more subcutaneous osteotomies were performed, in 28 cases cuneiform osteotomies were done, and in 12 instances osteoclasis was used. Of the 428 cases, 345 were discharged cured, 31 improved and 1, which was not rhachitic, but a badly united fracture of the tibia, unimproved.

For several years the subcutaneous method has practically always been employed; the cuneiform operation having been abandoned in these cases, even in anterior curves. When the bone is divided by a simple section and allowed to gape apart after correction the space fills up with new bone, and a good result is more certainly and safely attained than when a wedge is removed. It will rarely be necessary to divide the bone at more than one point.

Undoubtedly complications have arisen in connection with this operation, but I have observed none in my own practice, and have been unable to find any record or to hear of any mishaps of any consequence in the experience of the operators at the Hospital for the Ruptured and Crippled in the last ten years.

Mr. Edmond Owen called attention at the meeting of the British Medical Association in 1899 to the subject of ununited fracture of the tibia and fibula in children, relating several cases where union had failed to occur after an ordinary fracture, and if it were not for a large experience to the contrary one might fear this accident after osteotomy. A search through the reports of the Hospital for the Ruptured and Crippled for the last ten years shows but two operations for ununited fracture of the leg bones, both of the tibia, of which one followed a cuneiform osteotomy for a bad anterior tibial curve, the other a manual osteoclasis for a badly united fracture. Both finally united. Two other cases of ununited fracture following cuneiform osteotomy of the tibia in a girl of 3 and a girl of 13 have been observed by the writer. I have been unable to discover in this service a single case of this accident following subcutaneous osteotomy for knock-knees or bow-legs.

This experience agrees, so far as I am informed, with that of the principal operators for these deformities in this country.
CONCLUSIONS.

We may therefore conclude that:

1. Subcutaneous osteotomy of the shaft is a safe and certain operation for the correction of knock-knees and bow-legs:

2 . It is nearly always to be preferred to cuneiform osteotomy, osteotomy into the joint (for knock-knees) or operation by the open method.

3. The risk of non-union of bone or other accidents, with asepsis and an experienced operator, are slight, and not increased in rickety subjects.

4. The commonest errors are imperfect correction, especially failure to overcome inversion of the feet, and relapse from improper dressings, or from operating before the subsidence of the rhachitic process.

5. Plaster of paris makes an excellent splint, completely controlling the position of the fragments when properly applied. Plaster or other splints should be worn for some months.

6. Subcutaneous osteotomy may be likened to subcutaneous tenotomy:

a. In its simplicity, certainty and freedom from complications.

$b$. In the advisability of correction of the deformity at the time of operation, in the facility with which union occurs and gapes are filled up, in the importance of careful and intelligent postoperative treatment, and in the permissibility of locomotion before firm union has occurred, provided the parts are perfectly controlled by splints.

Each is indicated when the structures involved can not be permanently corrected by stretching in a reasonable time, and both mark a distinct advance in the treatment of certain deformities and are worthy of wider recognition.

\section{TREPHINING FOR BRAIN TUMORS; REPORT OF TWO SUCCESSFUL CASES-ONE OF NINE YEARS.* \\ JOSEPH RANSOHOFF, M.D., F.R.C.S. \\ PROFESSOR PRINCIRLES OF SURGERY, UNIVERSITY OF CINCINNATI. CINCINNATI.}

Operable brain tumors are rare. Only 6 per cent. of all cases are suitable for operation. In a recent compilation by Haas ${ }^{1} 122$ were tabulated. Permanent successes after operation are rare, even in the experience of such masters in this special field as Horsley, McEwen, Bergman and Keen. Therefore, every case carried to a successful issue is worthy of record. While I have trephined eight times for a supposed neoplasm, I was but twice rewarded by success. In a third case, a syphilitic thickening of the dura equivalent to a tumor formation was found. As a success from a diagnostic standpoint it is equivalent to a successful trephining for tumor. In each of these cases the tumor was in the psycho-motor area, thus again emphasizing the fact that only when in this limited field can brain tumors be recognized with any degree of certainty. My first case was operated on June 28, 1893. After nine years the patient continues well except for a slight paresis of the left extremities and epileptic seizures at intervals of three or four months. The case was reported by Dr. H. H. Hoppe. ${ }^{2}$ The following is a short history:

CASE 1.-A. L., aged 32 years, admitted to the Cincinnati Hospital, June 1, 1893. Family history negative; patient

* Read at the Fifty-third Annual Meeting of the American Medical Association, In the Section on Surgery and Anatomy, and approved for publication by the Executive Comat
Walker, A. J. Ochsner and DeForest Wllard. 